\title{
Full-field pulsed magnetophotoelasticity - experimental implementation
}

\author{
S Gibson ${ }^{1 *}$, G W Jewell ${ }^{2}$, and R A Tomlinson ${ }^{1}$ \\ ${ }^{1}$ Department of Mechanical Engineering, University of Sheffield, Sheffield, UK \\ ${ }^{2}$ Department of Electronic and Electrical Engineering, University of Sheffield, Sheffield, UK
}

The manuscript was received on 18 November 2004 and was accepted after revision for publication on 26 October 2005.

DOI: 10.1243/03093247JSA93

\begin{abstract}
This paper contains a description of the experimental procedure employed when using a pulsed magnetopolariscope (PMP) and some initial full-field through-thickness measurements of the stress distribution present in samples containing three-dimensional stresses. The instrument uses the theory of magnetophotoelasticity (MPE), which is an experimental stress analysis technique that involves the application of a magnetic field to a birefringent model within a polariscope. MPE was developed for through-thickness stress measurement where the integrated through-thickness birefringent measurement disguises the actual stress distribution. The technique is used mainly in toughened glass, where the through-thickness distribution can reduce its overall strength and so its determination is important.

To date, MPE has provided a single-point two-dimensional through-thickness measurement and the analysis time is prohibitive for the investigation of an area that may contain high localized stresses. The pulsed magnetopolariscope (PMP) has been designed to enable the application of full-field three-dimensional MPE, described in a companion paper. Using a proof-of-concept PMP, several experimental measurements were made; these were promising and demonstrate the potential of the new instrument. Further development of this technique presents several exciting possibilities, including a tool for the measurement of the distribution of the principal stress difference seen in a general three-dimensional model.
\end{abstract}

Keywords: three-dimensional photoelasticity, residual stress measurement, toughened glass

\section{INTRODUCTION}

The through-thickness stress distribution seen in toughened glass is of interest to many glass manufacturers because of the effect that this profile can have on the strength of glass. The technology available to measure this through-thickness profile is limited.

Transmission photoelastic measurements are routinely made in glass using a grey field polariscope [1], poleidoscope [2], and compensation tech-

\footnotetext{
* Corresponding author: Department of Mechanical Engineering, University of Sheffield, Mappin Building, Mappin Street, Sheffield S1 3JD, UK. email: sarah.gibson@sheffield.ac.uk
}

niques (e.g. Babinet and Sénarmount compensation). These techniques make two-dimensional photoelastic measurements and are useful but do not measure the through-thickness stress profile. Glass manufacturers also use a grazing angle surface polarizer (GASP) and a differential surface refractometer (DSR), but these only measure the stresses on one surface of the glass [3]. Other researchers have shown that scattered-light photoelasticity has great potential for the measurement of these through-thickness stresses, but it is the authors' understanding that these measurements are extremely time consuming [4].

Recently there has been much development of integrated photoelastic techniques, which are 
relevant to the measurement of the through-thickness stress distribution in glass. Integrated photoelasticity is a three-dimensional photoelastic technique that involves the collection of three characteristic parameters; these are measured through a birefringent model that contains a three-dimensional stress system. These characteristic parameters are functions of the integrated photoelastic effect which the model exhibits when light travels through it. Measurement of the characteristic parameters alone is often not sufficient to determine the stress distribution present through the thickness of the model, so more information in the form of an additional variable is required. This variable can be added by varying the viewing angle $[\mathbf{5}, \mathbf{6}]$, the load on the model $[\mathbf{7}, \mathbf{8}]$, the electric field [9], or the magnetic field [10] over the model. Assumptions can be made about the shape of the stress profile and these can reduce the amount of experimental data needed for its determination.

The combination of a magnetic field with integrated photoelasticity produces a technique known as magnetophotoelasticity (MPE) and this seems to provide the most suitable technique to meet the requirements of the glass industry. MPE was proposed by Aben [10] and developed by Clarke et al. $[11,12]$. It uses a magnetic field applied through the thickness of a birefringent component within a polariscope. The magnetic field causes rotation of the light vector as it travels through the sample; this phenomenon is known as the Faraday effect. If a loaded body is examined within a magnetic field using a polariscope, the final effect viewed through the apparatus is a combination of birefringence and the Faraday rotation within the sample. The photoelastic characteristic parameters (the characteristic retardation and the two characteristic directions [13]) are determined at the point of interest with and without the magnetic field. These values can then be used in an algorithm, utilizing an assumed stress profile, to determine the stress distribution present through the sample thickness [12]. Previously successful work on MPE was performed at Pilkington plc using a single-point magnetopolariscope, but tedious measurements have seen the instrument become redundant. The authors have designed a new instrument, the pulsed magnetopolariscope (PMP), which enables non-destructive full-field magnetophotoelastic measurement. This has been described more fully in a companion paper [14] and is described briefly in section 2 . This paper contains some examples of experimental measurements made with a proof-of-concept PMP which show the potential of the technique.

\section{THE APPARATUS OF A PROPOSED PULSED MAGNETOPOLARISCOPE}

The PMP has been suggested for the purpose of replacing a single-point magnetopolariscope and both are described in the companion paper [14]. The proposed PMP would have several advantages over the single-point magnetopolariscope, including an increased magnetic flux density and an increased field of view, resulting in a faster analysis of larger areas. It would consist of a pair of split 'air-cored' solenoid coils and a polariscope arranged around them. This arrangement would allow a complete sample to be positioned between the coils and an area to be examined. A capacitor-discharge magnetizer would be used to create a high intensity pulsed magnetic field lasting a few milliseconds within the bores of the coils and so through the sample [15]. A high-speed video camera would record the change in intensity of the light travelling through the sample caused by the magnetic pulse and the sample's birefringence.

\section{INTEGRATED PHOTOELASTICITY AND FOURIER POLARIMETRY}

In a general three-dimensional photoelastic body, the magnitude and direction of the principal stress difference varies continuously along the light path. The body can be represented by an optically equivalent model made up of a linear retarder and a pure rotator. Aben $[\mathbf{9}, \mathbf{1 3}]$ described this model in terms of three 'characteristic parameters', the characteristic retardation, $\Delta$, the primary characteristic direction, $\theta$, and the secondary characteristic direction, $\theta+\alpha$. The rotator angle, $\alpha$, is named the characteristic angle. In magnetophotoelasticity the characteristic parameters are determined through experiment, with and without the application of a magnetic field. These parameters are used with a set of algorithms that contain assumptions about the shape of the through-thickness distribution of stress to find the principal stress difference along the light path.

The characteristic parameters can be measured manually using a point-by-point technique. However, in order to implement three-dimensional MPE it is necessary to make a full-field automated collection of the characteristic parameters. Several research groups are working in the area of integrated photoelastic techniques and these include phase-shifting [16], Fourier polarimetry [17], and phase-stepping 
[18]. To make full-field MPE measurements a Fourier polarimetry method was chosen because, to date, it has produced the most accurate results. The accurate measurement of the characteristic parameters is especially important in glass, where these parameters are small and the Faraday rotation is limited by the strength of the magnetic field.

The Fourier polarimetry method used by the authors is based on the method used by Berezhna et al. [17]. In a PMP the polariscope (see Fig. 1) would be positioned around the solenoid coils. The polarizer and analyser would be rotated to discrete positions during a $360^{\circ}$ revolution of the polarizer. This is usually 36 or 72 positions, and at each position an image can be captured (note that actually a series of images can be captured during the pulse of the magnet when using a high-speed camera, and a frame at a particular flux density can be selected [14]). The analyser should be rotated to an angle three times the angle of the polarizer, to enable the analyser to rotate three times during one rotation of the polarizer. The intensity images then recorded can be considered to be one period of an infinite signal and as a result can be represented using a Fourier series. A program was written in Matlab which uses a Fourier transform and the intensity images to find the Fourier coefficients of the Fourier series. Using the Stokes vector and Mueller matrix representations the equations that relate the Fourier coefficients to the characteristic parameters can be found. The characteristic parameters in terms of the Fourier coefficients $a_{n}$ and $b_{n}$ are shown in the following equations and are derived in Appendix 2

$$
\begin{aligned}
& \alpha=\frac{360}{4 \pi} \arctan \left(\frac{b_{4}}{a_{4}}\right) \\
& \theta=\frac{360}{8 \pi}\left[\arctan \left(\frac{b_{8}}{a_{8}}\right) \pm \arctan \left(\frac{b_{4}}{a_{4}}\right)\right] \\
& \Delta=\frac{4 \times 360}{2 \pi} \arctan \left[\sqrt[4]{\frac{a_{8}^{2}+b_{8}^{2}}{a_{4}^{2}+b_{4}^{2}}}\right]
\end{aligned}
$$

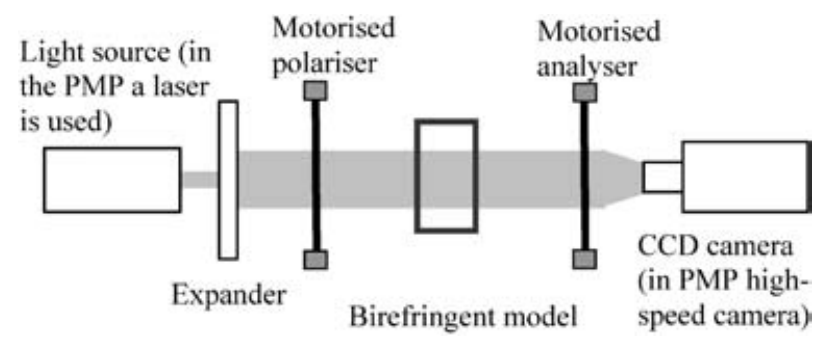

Fig. 1 Schematic showing the apparatus used for Fourier polarimetry which can be combined with a split solenoid coil to make a PMP
Now the experimental Fourier coefficients can be manipulated using equations (1) to (3) to find the experimental characteristic parameters.

With the novel combination of full-field Fourier polarimetry and a PMP, full-field maps of the characteristic parameters at selected magnetic flux densities can be collected. The principal stress difference may be obtained from these characteristic parameters, as discussed in section 4.3.

\section{EXPERIMENTS}

For the purposes of this paper a proof-of-concept instrument has been built. The proof-of-concept PMP [14] differs from an idealized device (discussed in section 2) because it contains an existing generalpurpose single solenoid coil and magnetizer unit (currently used for magnetizing small permanent magnet components for prototype machines and actuators). Therefore, the glass sample has to be sectioned in order to fit inside a single solenoid coil. As described above, a split coil will enable nondestructive testing. The proof-of-concept PMP also contains a high-speed camera and a green light laser source with associated polarizers. The system is capable of analysing samples up to $70 \mathrm{~mm}$ in diameter, at peak magnetic fields of about $4.1 \mathrm{~T}$. The light passes through a polarizer and a removable quarter-wave plate and into the specimen placed within the bore of the solenoid coil. The light then passes through a second polarizer. The light intensity image produced during the magnetic pulse is then recorded using the high-speed camera. The frames captured are synchronized with the magnetic pulse, thus allowing a sequence of full-field frames to be captured at several magnetic flux densities within the $2.5 \mathrm{~ms}$ required for the magnetic pulse to reach its peak. A schematic of the instrument is shown in the companion paper [14] and a typical magnetic pulse is plotted on the second axis of Fig. 2 .

\subsection{Experiment 1: the Faraday rotation}

\subsubsection{Faraday rotation measurement using the PMP}

The Faraday rotation, $F$, may be calculated from

$$
F=V B t
$$

where $V$ is the Verdet constant (which is a function of the material and the light wavelength, $\lambda$ ), $B$ is the magnetic flux density, and $t$ is the thickness of the material. Therefore, by knowing the Verdet constant 


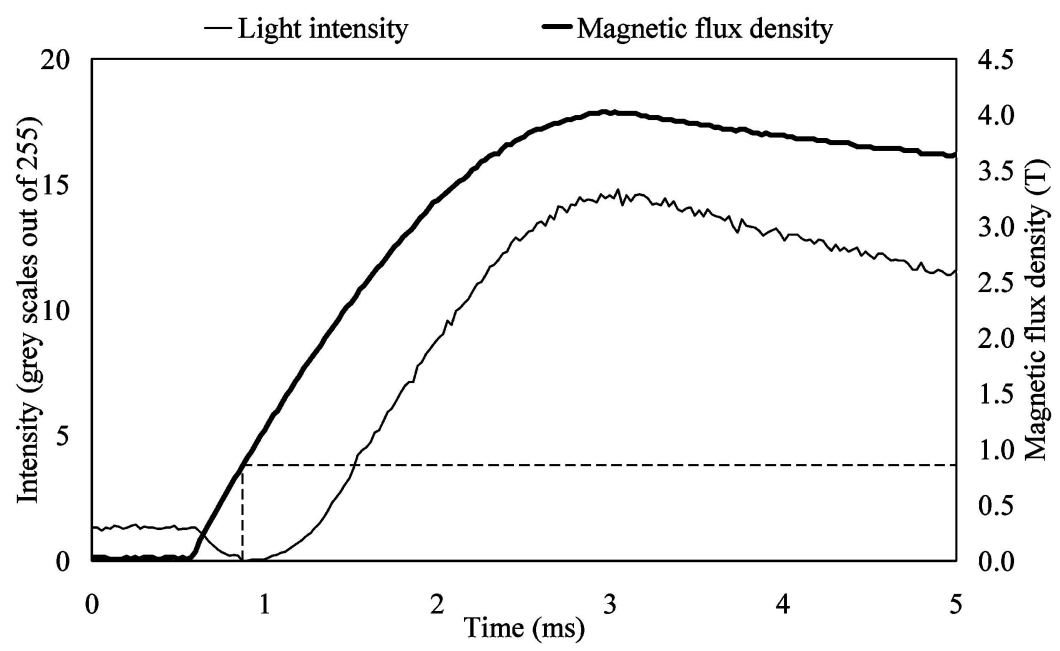

Fig. 2 Showing the intensity variation of a green laser during one pulse of the magnet. The polarizer was moved $1^{\circ}$ from its crossed position with the analyser

of a material, the Faraday rotation may be calculated for any magnetic flux density for light of a particular wavelength.

The aim of the first experiment was to measure the Faraday rotation of the light incident on a glass sample for a range of magnetic flux densities and therefore to find the Verdet constant for a given wavelength of light. This measurement may then be used in later calculations. A green light laser source of wavelength $\lambda=543 \mathrm{~nm}$ was used in the proof-ofconcept PMP and a stress-free sample of glass of thickness $2.13 \mathrm{~mm}$ was placed within the solenoid coil. Figure 2 shows a plot of the magnetic flux density over time and the corresponding intensity of the laser light when the polarizer was moved $1^{\circ}$ from its crossed position with the analyser. The magnetic flux density at which the light intensity is zero is the magnetic flux density at which the Faraday rotation is equal to the polariscope setting relative to its crossed position, in this case $F=1^{\circ}$ and $B=0.87 \mathrm{~T}$ (see Fig. 2). This measurement was repeated for a range of positions of the polarizer. The Faraday rotations were normalized with the thickness of the specimen and plotted against the corresponding magnetic flux density, $B$, as shown in Fig. 3. From equation (4) it can be seen that the slope of the line

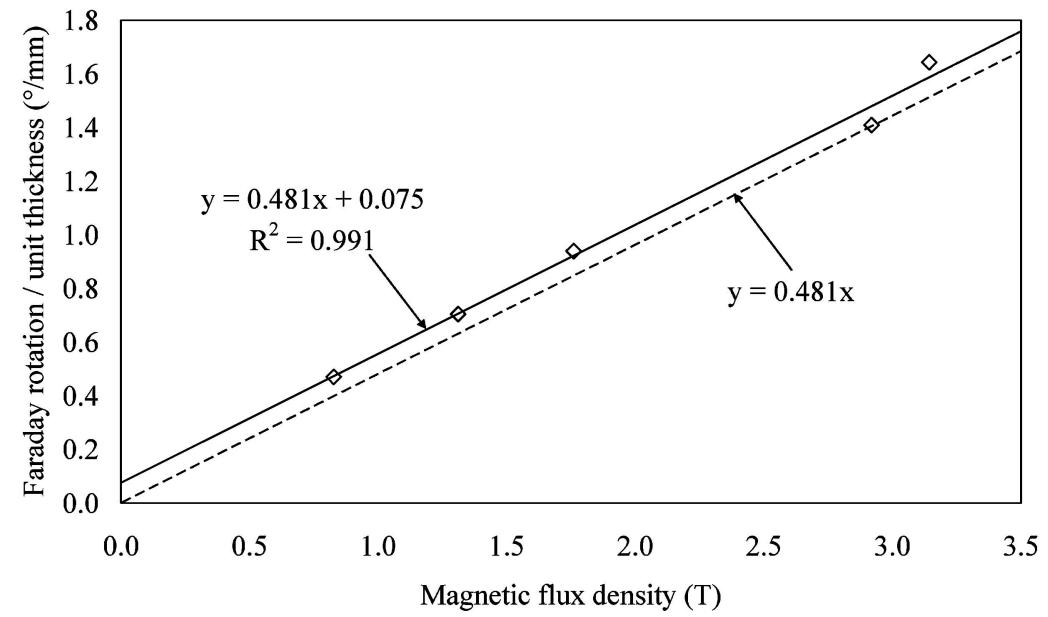

Fig. 3 Showing a plot of Faraday rotation/mm against magnetic flux density found using $2.13 \mathrm{~mm}$ thick stress-free glass sample and a green light laser source. The gradient of the graph is the Verdet constant. (The $R^{2}$ value shows how closely the experimental points fit the trend line. The closer this coefficient is to 1 the better the fit) 
fitted through the points is the Verdet constant of this glass for this wavelength of light. The linear regression line should pass through the origin of the graph; however, a constant inconsistency was apparent which was probably due to errors created during calibration of the magnetic field [14] and also to the effect of some residual stress within the sample. The slope of the line, the Verdet constant, was determined as $V=0.48 \mathrm{deg} / \mathrm{T} \mathrm{mm}$.

\subsubsection{Assessment of measurement accuracy}

A second measurement of the Verdet constant was made using a red light laser source, of wavelength $\lambda=633 \mathrm{~nm}$, incident on a $9.98 \mathrm{~mm}$ thick stress-free glass sample within the PMP. This measurement was made in order to compare the Verdet constant of the glass sample measured using the PMP with a measurement made on the same sample using the single-point instrument, which also uses a red light laser source of wavelength $\lambda=633 \mathrm{~nm}$. Figure 4 shows the Faraday rotation $/ \mathrm{mm}$ plotted against the magnetic flux density for the two instruments. It can be seen that the magnetic fields in the two magnetopolariscopes are opposite in sign. Again the line fitted to the data points found using the PMP showed a constant inconsistency and a correction factor was added to the magnetic flux density, shown by the black dotted line. It is notable that the fit of the line is extremely good with a correlation coefficient, $R^{2}=0.998$. This indicates that the measurement of the Verdet constant with the PMP is reliable at a range of magnetic flux densities. The Verdet constants calculated with the PMP and the single-point instrument were 0.262 and $0.253 \mathrm{deg} / \mathrm{T} \mathrm{mm}$ respectively, a difference of $0.009 \mathrm{deg} / \mathrm{T} \mathrm{mm}$.

To consider the effect of this inconsistency on the measured principal stress difference, consider a sample $9.98 \mathrm{~mm}$ thick as discussed above, but with an unknown through-thickness stress distribution. If a $4 \mathrm{~T}$ magnetic field travels through the sample and creates within the sample $15^{\circ}$ of Faraday rotation in the light vector, the measured Faraday rotation may differ from the rotation measured in the single-point polariscope by $0.36^{\circ}$ (i.e. $0.009 \mathrm{deg} / \mathrm{T} \mathrm{mm} \times 4 \mathrm{~T} \times$ $9.98 \mathrm{~mm}$ ). The range of solutions possible (solution maps or nomogram) for this glass sample with a Faraday rotation of $15^{\circ}$ was compared to the same problem but with $15.36^{\circ}$ of Faraday rotation. The difference between the two solution maps varies over the area from a minimum of 0.001 per cent and maximum of 6.68 per cent in the characteristic retardation and 0.09 to 2.43 per cent in the characteristic angle. When there is an appreciable amount of stress present in a glass sample, this represents a negligible effect on the obtained principal stress difference.

\subsection{Experiment 2: stress frozen beam in bending}

\subsubsection{Introduction}

An experiment was undertaken in order to validate the use of the PMP and to assess its accuracy. A beam in bending was chosen since this profile is

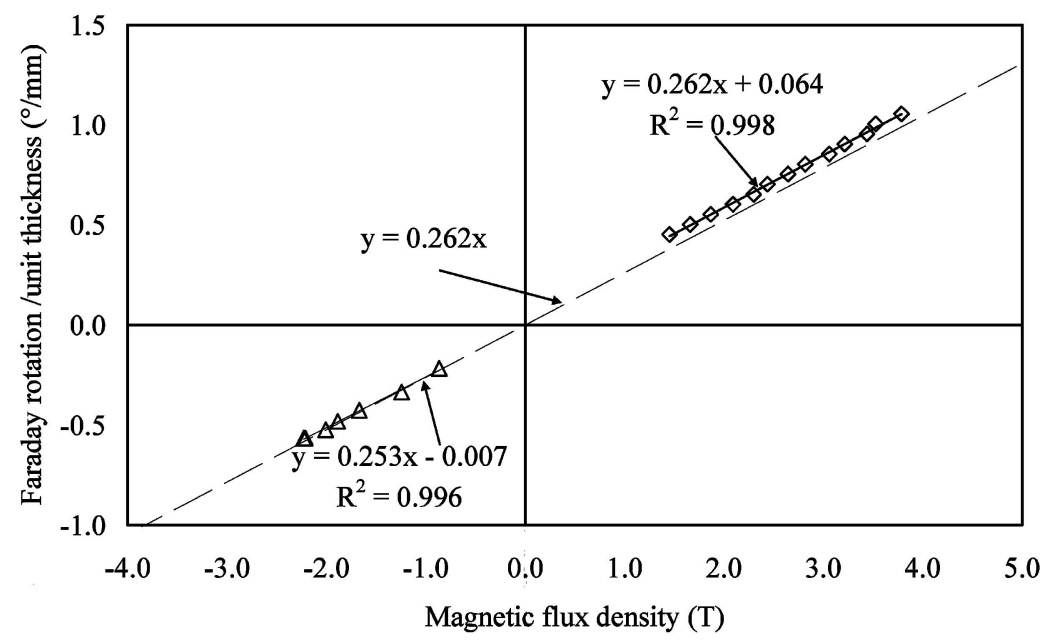

Fig. 4 Showing a comparison between the Faraday rotation $/ \mathrm{mm}$ measured in stress-free glass using both the single-point magnetopolariscope (triangles) and the pulsed magnetopolariscope (diamonds). The gradient of the lines is the Verdet constant. (The $R^{2}$ value shows how closely the experimental points fit the trend line. The closer this coefficient is to 1 the better the fit) 
easy to create and the integrated photoelastic effects generated by the compressive and tensile stresses cancel out each other along the light path, thus disguising the stress distribution.

\subsubsection{Experiment}

Two beams made from epoxy resin (PSM-9 from Vishay Measurements Group, Inc.) with a crosssectional area of $10 \mathrm{~mm} \times 6.54 \mathrm{~mm}$ were loaded in four-point bending. Surface strains of 400 and 800 microstrain were applied to beams A and B respectively and these beams were then stress-frozen. After stress-freezing, the bending strains applied correspond to a final surface stress of 106 and $212 \mathrm{~N} / \mathrm{mm}^{2}$ for beams A and B respectively. The specimens were then positioned within the solenoid coil of the PMP using a plastic sample holder, as shown in Fig. 5. The characteristic parameters of the beams with an applied magnetic field of 0 and $3.2 \mathrm{~T}$ were collected. To achieve this, 72 frames, each of $64 \times 64$ pixels in size and with a resolution of about 4.3 pixels $/ \mathrm{mm}$,

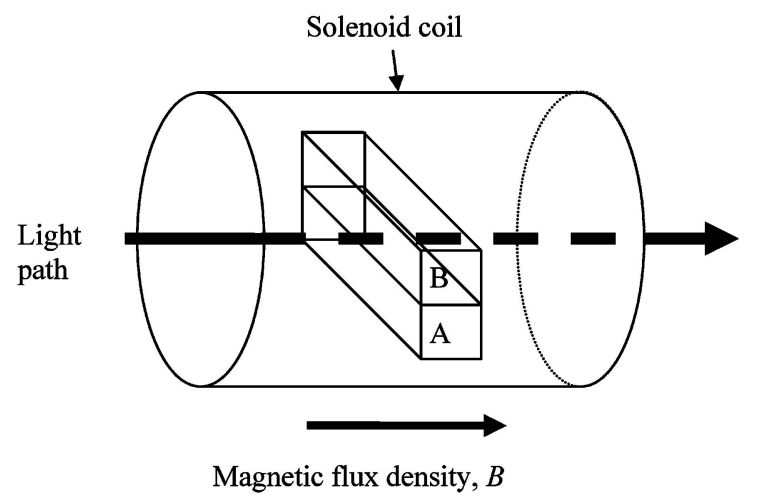

Fig. 5 A schematic showing two epoxy beams in the solenoid coil. Beam A was stress-frozen with $400 \mu \varepsilon$ and B was stress-frozen with $800 \mu \varepsilon$. The plane of bending of each beam is horizontal as shown were recorded at the different positions of the polarizer and analyser for both field strengths. Each of these frames was recorded during a separate pulse of the magnet. The Fourier polarimetry method was used to process the images to find the samples' characteristic parameters (see section 3 on Fourier polarimetry above) [17]. Maps of the characteristic angle and retardation were produced and an area of $10 \times 10$ pixels was selected from the same location, corresponding to the centre of the beams in both characteristic parameter maps. An average of these pixels was taken and the resulting two parameters for each beam are entered in the first and second rows of Table 1.

\subsubsection{Theoretical prediction using a nomogram}

In a situation of pure bending, when viewed in the direction shown in Fig. 5, the characteristic retardation in a beam should equal zero, since the integrated photoelastic effects generated by the compressive and tensile stresses cancel out each other. However, a positive value of characteristic retardation is apparent in the measurements (see Table 1). A standard polariscope was used to implement Tardy compensation, which confirmed that the characteristic retardation was greater than zero. A solution graph or nomogram was plotted for the chosen Faraday rotation and is shown in Fig. 6. This nomogram is for a beam in bending with a superimposed membrane stress and $15.43^{\circ}$ Faraday rotation. The dashed lines are contours of constant characteristic angles and the solid contours show constant characteristic retardation. To use this nomogram the bending and membrane principal stress difference within the sample can be read off the $x$ and $y$ coordinates of the point where the experimentally measured characteristic angle and retardation coincide on the nomogram. Figure 6 was created using a general method described in the appendix of Clarke et al.

Table 1 Showing a comparison between the experimentally and theoretically measured profiles found in beams A and $\mathrm{B}$

\begin{tabular}{|c|c|c|c|}
\hline & Units & Beam A & Beam B \\
\hline Characteristic angle & $\operatorname{deg}$ & 11.97 & 9.58 \\
\hline Characteristic retardation & deg & 132.0 & 136.4 \\
\hline $\begin{array}{l}\text { Experimental surface principal stress difference produced by a bending moment applied to the } \\
\text { beams read from the nomogram (Fig. 6) }\end{array}$ & $\mathrm{N} / \mathrm{mm}^{2}$ & 106.4 & 208.8 \\
\hline Theoretical surface principal stress difference produced by a bending moment applied to the beams & $\mathrm{N} / \mathrm{mm}^{2}$ & 106.2 & 212.5 \\
\hline Membrane stress read from the nomogram (Fig. 6) & $\mathrm{N} / \mathrm{mm}^{2}$ & 9.30 & 9.60 \\
\hline Membrane stress measured using Tardy compensation & $\mathrm{N} / \mathrm{mm}^{2}$ & 9.55 & 9.24 \\
\hline $\begin{array}{l}\text { Maximum difference between the experimentally and theoretically measured profiles of principal } \\
\text { stress difference }\end{array}$ & $\mathrm{N} / \mathrm{mm}^{2}$ & 0.47 & 3.99 \\
\hline
\end{tabular}




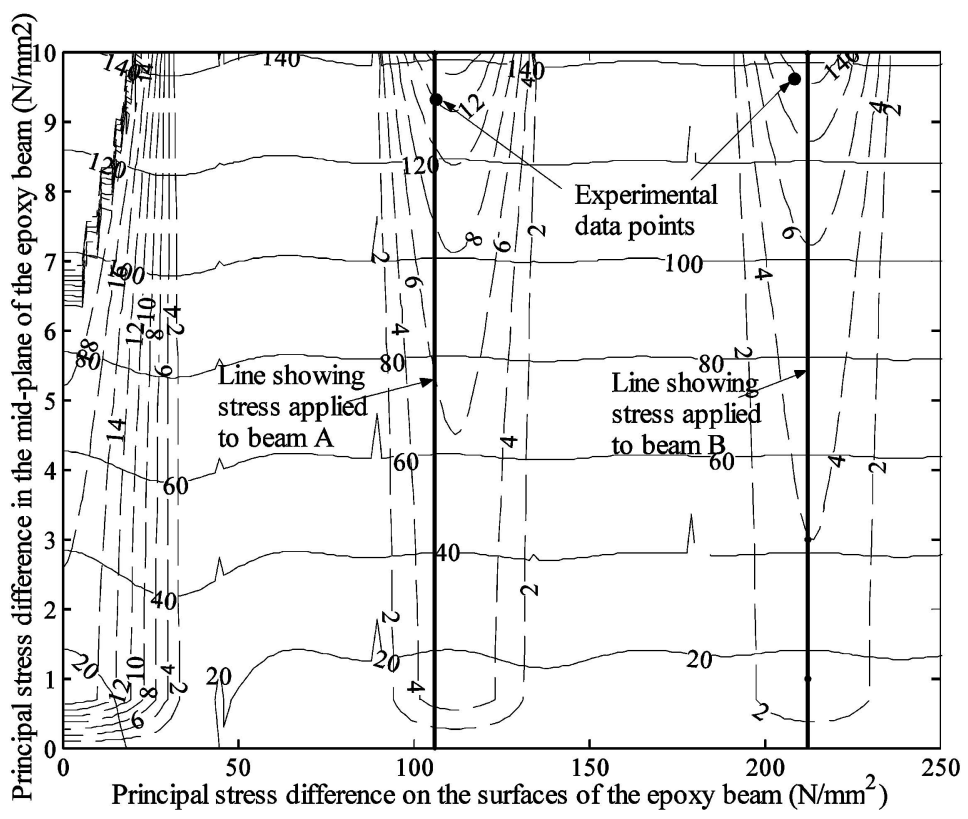

Fig. 6 Showing a nomogram for the principal stress distribution of a beam in bending with a superimposed membrane stress subject to a Faraday rotation of $15.43^{\circ}$. The dashed contour shows lines of constant characteristic angle and the solid lines constant characteristic retardation. The thick black lines show the bending principal stress difference applied to the beams during the stress-freezing process

[12] and for further information this should be consulted. Unfortunately, the nomogram is a repeating one which produces several solutions for a set of experimental measurements. However, as the position of the solution was roughly known because of the applied bending stress, this value was used as a starting point to search for a stress distribution that would create the characteristic parameters measured experimentally.

\subsubsection{Results}

An area representing approximately $5.4 \mathrm{~mm}^{2}$ and containing $10 \times 10$ pixels was selected from the data maps of the characteristic retardation and angle. The averages of the characteristic parameters in these areas are shown in Table 1. Using the known applied bending stress as the start point, the nomogram was searched for the position of the measured characteristic parameters. The profile of principal stress difference was then read from the axes of the nomogram. This profile is described through the superposition of a bending stress and a membrane stress; these values are entered into the third and fifth rows of Table 1. These values were compared with the applied bending stress and the through-thickness retardation was measured using Tardy compen- sation, which are shown in the fourth and sixth rows of Table 1. The differences in the resulting stress profiles are given in the seventh row of Table 1; this shows that the greatest difference is approximately $4 \mathrm{~N} / \mathrm{mm}^{2}$ or 2 per cent, which is insignificant.

\subsection{Experiment 3: full-field three-dimensional residual stress measurement in glass}

An experiment was conducted to determine the full-field through-thickness principal stress difference in a $9.98 \mathrm{~mm}$ thick, disc-shaped toughened glass sample. The sample was positioned within the solenoid coil and the high-speed camera recorded a sequence of frames during a pulse of the magnetic field. The series of frames were downloaded on to a computer and then matched to the pulse of the magnetic field. From this series two frames which were captured at flux densities closest to the levels of 0 and $3.2 \mathrm{~T}$ were selected. Fourier polarimetry was implemented with 36 orientations of the polarizer and analyser, and through the selected images, maps of the characteristic parameters at these two magnetic field strengths were found. In order to determine the full-field principal stress difference from these data maps, a genetic algorithm [19] was utilized. 
A program used to create a nomogram for the distribution of the principal stress difference approximated by a parabola with superimposed bending was adapted into a genetic algorithm. This genetic algorithm searches a range of principal stress difference profiles to find the distribution that could create the theoretical characteristic parameters which matched the experimentally measured characteristic parameters. The distribution consisting of a parabola and a superimposed bending stress is found and is defined fully using the three coefficients of a quadratic equation. The genetic algorithm was created using a Matlab ${ }^{\odot}$ toolbox; this particular method was chosen because it was fast to execute. Previously this search for the correct principal stress difference solution was implemented by an operator who used iterative inputs. This procedure was time consuming, especially for the analysis of an area [12]. The genetic algorithm is a faster and more reliable search strategy when compared to the manual computer aided search. This simple approach was quick to implement but could be improved considerably in terms of the solution time and so other methods will be considered for future work. Figure 7 shows full-field measurements of the through-thickness principal stress difference in the $9.98 \mathrm{~mm}$ thick glass sample. From these maps a distribution consisting of a superimposed parabola and a bending stress can be

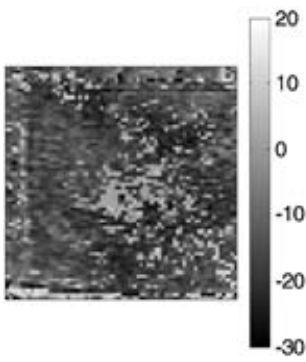

a) Principal stress difference on front surface $\mathrm{N} / \mathrm{mm}^{2}$

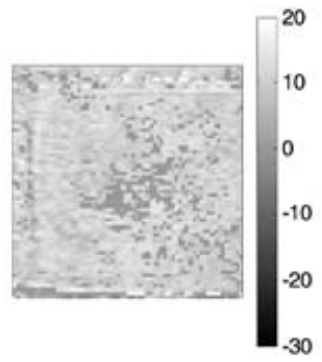

b) Principal stress difference in the mid-plane $\mathrm{N} / \mathrm{mm}^{2}$

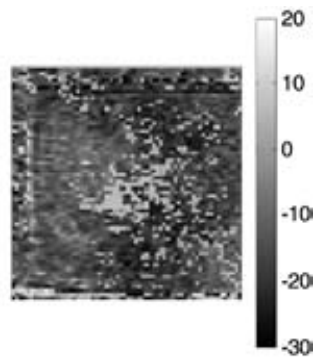

c) Principal stress

difference on the

back surface $\mathrm{N} / \mathrm{mm}^{2}$

Fig. 7 Showing the principal stress difference on the two surfaces (a) and (c) and in the mid-plane (b) of a glass sample

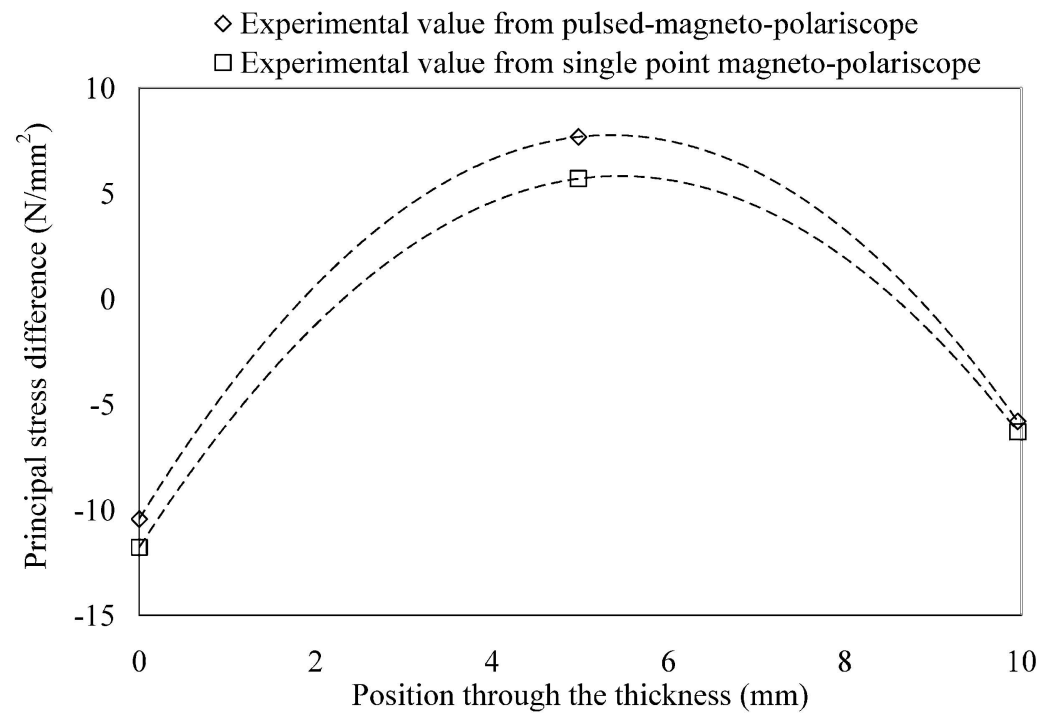

Fig. 8 Showing the principal stress difference through the thickness of a glass sample. The two plots are results taken from the PMP and from the single-point magnetopolariscope 
plotted and an example of this, for a single point in the field, is shown in Fig. 8. In Fig. 8 a measurement made using the single-point magnetopolariscope is also plotted to make a comparison between the instruments. The two readings show close agreement, but the maximum difference seen is approximately $\pm 2 \mathrm{~N} / \mathrm{mm}^{2}$, a discrepancy of up to 25 per cent.

\section{DISCUSSION}

This proof-of-concept apparatus has several advantages over the older equipment developed [12]. These advantages include a 71 per cent increase in the magnetic field (this could be further increased with a larger magnetizer unit), a larger field of view, and more rapid measurements. The apparatus also allows a three-dimensional stress measurement, unlike the two-dimensional single-point magnetopolariscope. The increase in magnetic field strength, and hence the Faraday rotation, will reduce the sensitivity of the method to experimental errors. This is especially beneficial in thinner glasses where the Faraday rotation is small. Preliminary stress measurements in a glass disc (Experiment 3 ) show a $\pm 2 \mathrm{~N} / \mathrm{mm}^{2}$ agreement between measurements taken using the single-point magnetopolariscope and the new PMP. This difference is up to 25 per cent of the measured value and while this is large it still shows the promise of the PMP. The stress measured in the sample was low and a higher toughening stress would make the measurement less susceptible to error. The accuracy of the instrument could be improved through development of a customdesigned light source, but the PMP's main limitation is the accuracy at which full-field integrated photoelasticity can be implemented.

In Experiment 1, measurement of the Faraday rotation at $4 \mathrm{~T}$ showed a $\pm 0.036 \mathrm{deg} / \mathrm{mm}$ agreement between measurements taken using the single-point magnetopolariscope and the proof-of-concept PMP. This represents a negligible effect on the obtained principal stresses. In Experiment 2, comparison of the theory to the measured profiles in two epoxy beams in bending gave a maximum discrepancy of approximately $\pm 4 \mathrm{~N} / \mathrm{mm}^{2}$, some 2 per cent of the surface principal stress difference. The limitation of this measurement was that it had to use the applied bending stress as an initial starting point to search for the stress distribution solution. However, the analysis of any other known and interesting threedimensional stress distribution is difficult, and the lack of other equipment to measure a more general distribution and so validate the instrument is the main motivation for this work. The development of the multimagnetophotoelastic method discussed in the companion paper [14] could be used for a more definite validation of the equipment because of its ability to measure a general stress distribution, thus extending the range of models that can be created and examined.

Automated processing of the characteristic parameters to find the distribution of the principal stress difference was needed in order to develop a successful three-dimensional fully automated MPE technique. A genetic algorithm was used to search for the distribution of the principal stress difference that matched the experimentally measured characteristic parameters found in toughened glass [20]. This search assumed that the shape of the principal stress difference was a distribution consisting of a superimposed parabola and a bending stress. This algorithm not only speeds up the process of finding the principal stress difference from the characteristic parameters, when compared to the previous highly interactive manual computer search method, but also gives a more extensive and more reliable search method by removing human error. The genetic algorithm always finds the solution and the method is as sensitive as the manual search method; any further sensitivity would be meaningless because of the resolution that can be obtained. However, the process is slow for large areas and so further development is required. A repeatability test was performed on the genetic algorithm. The distribution of the principal stress difference found through repeated processing of several sets of characteristic data has a standard deviation of $0.21 \mathrm{~N} / \mathrm{mm}^{2}$. This is more than adequate for the purposes described here.

Currently the collection of 72 sequences of images takes 12 hours. This is because the high currents produced by the magnetizer heat the solenoid coil. For safety reasons a pulse was therefore made only every 10 minutes. In industry this type of magnet is cooled and can therefore be used repeatedly. Charging the magnetizer takes only a few seconds and the discharge may be performed in milliseconds. Using purpose-built split solenoid coils and a higher specification camera to create a prototype PMP of the type described in section 2 , it has been estimated that the collection of 144 images would take approximately 12 minutes (see Table 2). This speed is about 60 times faster than the collection of full-field experimental data using the proof-of-concept instrument. This improvement, as can be seen in the first row of Table 2, is due mainly to the reduction in the time 
Table 2 Time study for data collection using the proof-of-concept and the prototype PMP

\begin{tabular}{lll}
\hline Action per image & Proof-of-concept PMP & Prototype PMP \\
\hline Charging and cooling the magnetizer (s) & 610 & 10 \\
Pulse of magnetic field (s) & 0.025 & 0.030 \\
Downloading images (s) & 0.50 & 0.16 \\
Total time to collect 72 images (h) & 12.21 & 0.20 \\
\hline
\end{tabular}

necessary to cool the solenoid coil. Some improvement can be made in reducing the time taken to download images from the camera to a computer but the most significant improvement gained through use of a higher specification camera would be the increased resolution and image quality. A prototype PMP would collect full-field data four times faster than the 50 minutes required to make only one measurement using the single-point magnetopolariscope, and this shows the real benefit of the PMP to industry.

\section{CONCLUSIONS}

A proof-of-concept PMP has been designed, assembled, and validated. Full-field maps of the principal stress difference over an area and through the thickness of glass samples have been generated using this novel apparatus. There are still many areas of the apparatus that can be improved and still more testing is required to assess the true potential, accuracy, and repeatability of the instrument. These initial tests have proved the validity of continuing with further development and testing.

This research has produced a novel apparatus capable of rapid evaluation of the principal stress difference within a sample of float glass. The main advantages of the novel proof-of-concept instrument are:

(a) an analysis area over 300 times larger (no upper limit has been identified);

(b) 71 per cent higher magnetic flux density (possibility of generating fields up to $20 \mathrm{~T}$ );

(c) more rapid data collection;

(d) homogeneous magnetic flux density over the analysis volume;

(e) possibility of determining a general throughthickness distribution of the principal stress difference with no $a$ priori knowledge.

\section{ACKNOWLEDGEMENTS}

The authors would like to thank Andrew Race and John Wilkinson for help with the triggering system, Richard Kay for building some of the apparatus, Pilkington plc and EPSRC for sponsorship of this project, and the EPSRC Instrument Loan Pool for the loan of the high-speed video camera.

\section{REFERENCES}

1 Calvert, G., Lesniak, J., and Honlet, M. Applications of modern automated photoelasticity to industrial problems. Insight, 2002, 44(4), 1-4.

2 Patterson, E. A. Digital photoelasticity: principles, practice and potential. Strain, 2002, 38, 27-39.

3 Khaleel, M. A., Woods, J. L., and Shepard, C. L. Surface stress measurement on automobile windshields. Glass Technol., 2001, 42(2), 49-53.

4 Hundhammer, I., Lenhart, A., Pontasch, D., and Weißmann, R. Stress measurement in transparent materials using scattered laser light. Glass Sci. Technol., 2002, 75, 236-242.

5 Hammer, H., Lionheart, W. R. B., and Tomlinson, R. A. Recent advances in tomographic photoelasticity. In CD-ROM Proceedings of the 2004 SEM X International Congress on Experimental and applied mechanics, Costa Mesa, California, 7-10 June 2004, Paper 33.

6 Errapartt, A., Aben, H., and Ainola, L. Photoelastic tomography in linear approximation. In CD-ROM Proceedings of the 2004 SEM X International Congress on Experimental and applied mechanics, Costa Mesa, California, 7-10 June 2004, Paper 47.

7 Tomlinson, R. A. and Patterson, E. A. On the feasibility of determining discrete photoelastic parameters using the multi-load method. J. Strain Analysis, 1999, 34(4), 295-299.

8 Keshavan, S. Y. The multi-load method of determining discrete system parameters in a loaded three-dimensional photoelastic model. J. Strain Analysis, 1994, 30(1), 3-8.

9 Aben, H. K. Integrated photoelasticity, 1979 (McGraw-Hill, New York). 
10 Aben, H. K. Magnetophotoelasticity - photoelasticity in a magnetic field. Expl Mechanics, 1970, 10, 97-105.

11 Clarke, G. P. Measurement of residual stress in glass, using magnetophotoelasticity. Thesis, University of Nottingham, October 1979.

12 Clarke, G. P., McKenzie, H. W., and Stanley, P. The magnetophotoelastic analysis of residual stresses in thermally toughened glass. Proc. R. Soc. London A, 1999, 455, 1149-1173.

13 Aben, H. K. Optical phenomena in photoelastic models by the rotation of principal axis. Expl Mechanics, 1966, 6, 13-22.

14 Gibson, S., Jewell, G. W., and Tomlinson, R. A. Fullfield pulsed magnetophotoelasticity: a description of the instrument. J. Strain Analysis, 2006, 41(2), 161-170.

15 Riley, C. D. The design of magnetizing fixtures and powder aligning systems for bonded $\mathrm{NdFeB}$ Permanent Magnets. Thesis, University of Sheffield, 1996.

16 Mangal, S. K. and Ramesh, K. Determination of characteristic parameters in integrated photoelasticity by phase-shifting techniques. Optics and Lasers in Engng, 1999, 31, 263-278.

17 Berezhna, S., Berezhnyy, I., and Takashi, M. Integrated photoelasticity through imaging Fourier polarimetry of an elliptic retarder. Appl. Optics, 2001, 40(5), 644-651.

18 Tomlinson, R. A. and Patterson, E. A. Measurement of Poincaré characteristic parameters using phase stepping. Expl Mechanics, 2002, 42(1), 43-50.

19 Pacey, M. N., James, M. N., and Patterson, E. A. A new photoelastic model for studying fatigue crack closure. Expl Mechanics, 2005, 45(1), 42-52.

20 Gibson, S. Experimental determination of the through-thickness residual stress distribution in glass. Thesis, University of Sheffield, May 2004.

\section{APPENDIX 1}

\section{Notation}

$a_{n}, b_{n} \quad$ Fourier coefficients (grey scales in rad)

$B \quad$ magnetic flux density (T)

$F \quad$ Faraday rotation (deg)

$t \quad$ thickness of the medium (mm)

$V \quad$ Verdet constant (deg/T mm)

$\alpha \quad$ characteristic angle (deg)

$\Delta \quad$ characteristic retardation (deg)

$\theta \quad$ primary characteristic direction (deg)

$\theta+\alpha \quad$ secondary characteristic direction (deg)

$\lambda \quad$ wavelength of light (nm)

\section{APPENDIX 2}

\section{Determination of the characteristic parameters using Fourier polarimetry}

$a_{n}, b_{n} \quad$ coefficients of a Fourier series (grey scale radians)

$I(r) \quad$ light intensity as a function of polarizer position $r$ (grey scale)

$\mathbf{M}_{\theta}(\delta) \quad$ Mueller matrix representing a birefringent model with principal stress direction $\theta$ and retardation $\delta$

$n$ number of harmonics used, i.e. $n=1,2,3, \ldots, \infty$

$\mathbf{P}_{\mathrm{A}} \quad$ Mueller matrix for a polarizer with its major axes at angle $\mathrm{A}$

$\mathbf{P}_{\mathrm{P}} \quad$ Mueller matrix for a polarizer with its major axes at angle $\mathrm{P}$

$r \quad$ rotational position of the polarizer (rad)

$\mathbf{R}_{\alpha} \quad$ Mueller matrix of a rotation matrix with rotation $\alpha$

$S_{\text {in }} \quad$ Stokes vector of randomly polarized light

$S_{\text {out }} \quad$ Stokes vector of the light out of the polariscope

$s_{0} \quad$ first element of the Stokes vector $S_{\text {out }}$

$\alpha \quad$ characteristic angle (rad)

$\Delta \quad$ characteristic retardation $=2 \delta(\mathrm{rad})$

$\theta \quad$ primary characteristic direction (rad)

The optical elements of the polariscope and model (in terms of the characteristic parameters) are described using Mueller matrices and Stokes vectors. The Stokes vector out of the model can be described as

$$
S_{\text {out }}=\mathbf{P}_{\mathrm{A}} \mathbf{R}_{\alpha} \mathbf{M}_{\theta}(\delta) \mathbf{P}_{\mathrm{P}} S_{\text {in }}
$$

where

$$
\begin{aligned}
& \boldsymbol{S}_{\mathrm{in}}=\left[\begin{array}{l}
1 \\
0 \\
0 \\
0
\end{array}\right]=\left[\begin{array}{l}
s_{0} \\
s_{1} \\
s_{2} \\
s_{3}
\end{array}\right] \\
& \mathbf{P}_{\mathrm{A}}=\frac{1}{2}\left[\begin{array}{cccc}
1 & \cos (2 A) & \sin (2 A) & 0 \\
\cos (2 A) & \cos ^{2}(2 A) & \sin (2 A) \cos (2 A) & 0 \\
\sin (2 A) & \sin (2 A) \cos (2 A) & \sin ^{2}(2 A) & 0 \\
0 & 0 & 0 & 0
\end{array}\right]
\end{aligned}
$$




$$
\mathbf{M}_{\theta}(\delta)=\left[\begin{array}{cccc}
1 & 0 & 0 & 0 \\
0 & \cos ^{2}(2 \theta)+\sin ^{2}(2 \theta) \cos (\delta) & {[1-\cos (\delta)] \sin (2 \theta) \cos (2 \theta)} & -\sin (2 \theta) \sin (\delta) \\
0 & {[1-\cos (\delta)] \sin (2 \theta) \cos (2 \theta)} & \sin ^{2}(2 \theta)+\cos ^{2}(2 \theta) \cos (\delta) & \cos (2 \theta) \sin (\delta) \\
0 & \sin (2 \theta) \sin (\delta) & -\cos (2 \theta) \sin (\delta) & \cos (\delta)
\end{array}\right]
$$

$$
\mathbf{R}_{\alpha}=\left[\begin{array}{cccc}
1 & 0 & 0 & 0 \\
0 & \cos (2 \alpha) & -\sin (2 \alpha) & 0 \\
0 & \sin (2 \alpha) & \cos (2 \alpha) & 0 \\
0 & 0 & 0 & 0
\end{array}\right]
$$

The intensity of the light emerging from the birefringent model can be described as

$$
I(r)=s_{0}
$$

Using a Fourier series, a periodic function can be represented by

$$
I(r)=\frac{a_{0}}{2}+\sum_{n=1}^{\infty}\left[\alpha_{n} \cos (n r)+b_{n} \sin (n r)\right]
$$

where the Fourier coefficients are

$$
\begin{aligned}
& a_{0}=\frac{2}{\pi} \int_{0}^{2 \pi} I(r) \mathrm{d} r \\
& a_{n}=\frac{2}{\pi} \int_{0}^{2 \pi} I(r) \cos (n r) \mathrm{d} r \\
& b_{n}=\frac{2}{\pi} \int_{0}^{2 \pi} I(r) \sin (n r) \mathrm{d} r
\end{aligned}
$$

By putting equation (10) into equations (12) to (14) some of the Fourier coefficients in terms of the characteristic parameters are obtained as

$$
\begin{aligned}
& a_{4}=\frac{1}{2} \cos ^{2} \frac{\delta}{2} \cos 2 \alpha \\
& a_{8}=\frac{1}{2} \sin ^{2} \frac{\delta}{2} \cos (2 \alpha+4 \theta) \\
& b_{4}=\frac{1}{2} \cos ^{2} \frac{\delta}{2} \sin 2 \alpha \\
& b_{8}=\frac{1}{2} \sin ^{2} \frac{\delta}{2} \sin (2 \alpha+4 \theta)
\end{aligned}
$$

These can be rearranged to find the characteristic parameters as

$$
\begin{aligned}
& \alpha=\frac{1}{2} \arctan \left(\frac{b_{4}}{a_{4}}\right) \\
& \theta=\frac{1}{4}\left[\arctan \left(\frac{b_{8}}{a_{8}}\right) \pm \arctan \left(\frac{b_{4}}{a_{4}}\right)\right] \\
& \Delta=2 \delta=4 \arctan \left(\sqrt[4]{\frac{a_{8}^{2}+b_{8}^{2}}{a_{4}^{2}+b_{4}^{2}}}\right)
\end{aligned}
$$

